Eixo Temático: Biologia Aplicada

\title{
ET-09-023 \\ RESPOSTA CARDIOVASCULAR AO ESTRESSE EMOCIONAL AGUDO DE RATOS SUBMETIDOS À SUPLEMENTAÇÃO ALIMENTAR RICA EM FRUTOSE
}

Breno Tenório Galvão Fonseca, Bianca Maria Vieira Vasconcelos, Priscila da Silva Guimarães

Setor de Fisiologia. Instituto de Ciências Biológicas e da Saúde. Universidade Federal de Alagoas.

http://dx.doi.org/10.21472/congrebio2016.et-09-023

\section{RESUMO}

O estresse é um fator de risco para desenvolvimento de doenças cardiovasculares em seres humanos e diversos estudos têm mostrado que a obesidade induz a uma resposta cardíaca exacerbada a exposição ao estresse. Em adição, o consumo elevado de frutose na atual sociedade, tem se mostrado um fator predisponente ao surgimento da síndrome metabólica, que por sua vez, oferece prejuízos a saúde do coração. Neste contexto, o objetivo deste estudo foi avaliar a capacidade de resposta cardiovascular ao estresse agudo de contenção e outras alterações fisiológicas em uma dieta rica em frutose (CEUA- UFAL 19/ 2015). Para isso, ratos wistar machos (7 semanas de idade) foram submetidos a 10 semanas de dieta rica em frutose (FF) ou água potável (grupo controle - CTL). Foram avaliados: a pressão arterial média (PAM), a frequência cardíaca (FC), a sensibilidade do controle barorreflexo da frequência cardíaca (BRS), a resposta cardiovascular ao estresse agudo de contenção, e o tônus simpático vascular por bloqueio farmacológico (prazosina, $0,1 \mathrm{mg} / \mathrm{kg}$ i.v). Os resultados foram analisados quanto à normalidade e variabilidade da amostragem. Diferenças entre os grupos foram analisadas utilizando o teste t Student $(\mathrm{p}<0.05)$. Os ratos FF mostraram um aumento significativo na PAM ( $F F=129,71 \pm 2,0$ vs CTL=117,14 $\pm 1,5 \mathrm{mmHg}$ ), e uma tendência ao aumento da FC (FF = 309,86 \pm 7,1 vs CTL $=287,29 \pm 8,7$ bpm; $\mathrm{p}=0.067$ ) comparado ao CTL. Como esperado, o grupo FF mostrou um prejuízo no BRS ( $\mathrm{FF}=1.11 \pm 0.08$ vs $\mathrm{CTL}=1.62 \pm 0.08 \mathrm{~ms} / \mathrm{mmHg}$ ). No que se refere à resposta cardiovascular ao estresse, o grupo $\mathrm{FF}$ mostrou uma resposta cardiovascular exacerbada, apresentando maiores variações de PAM (FF $=37,5 \pm 4,2$ vs CTL $=$ 29,0 \pm 3,6 mmHg) e de FC (FF = 150,3 $\pm 19,5$ vs CTL = 79,8 \pm 20 bpm) durante e logo após o estresse. Não houve diferença quanto ao tônus simpático vascular ( $>>0,05)$. Conclui-se que a suplementação alimentar crônica rica em frutose, além de promover aumento PAM e FC de repouso, e reduzir a sensibilidade do controle barorreflexo da FC, também contribui para uma resposta cardiovascular exacerbada ao estresse emocional moderado, conferindo a este um potencial fator de risco adicional ao desenvolvimento de doenças cardiovasculares. (FAPEAL).

Palavras-chave: Estresse; Síndrome Metabólica; Sistema Nervoso Autônomo.

\section{INTRODUÇÃO}

A síndrome metabólica (SMet) é um conjunto de fatores de risco, tais como resistência à insulina, hiperinsulinemia, hipertrigliceridemia, arteriosclerose e hipertensão arterial, que contribuem para aumentar as chances de doenças cardiovasculares, diabetes mellitus tipo 2 e doenças renais (ABDULLA, 2011).

Estudos realizados em humanos e em roedores indicam que o aumento da ingestão em longo prazo de frutose pode estar relacionada com o aparecimento de diferentes alterações cardiovasculares e metabólicas relacionadas à SMet. Esse aumento mundial na ingestão de frutose se deve principalmente a sua maior utilização na indústria alimentícia como xarope de 
milho rico em frutose, devido ao seu baixo custo e por conferir maior tempo de prateleira ao produto (TAPPY e LÊ, 2010). As principais alterações cardiovasculares observadas com o maior consumo de frutose são: aumento da pressão arterial média (PAM), prejuízo no controle barorreflexo da frequência cardíaca (FC) e disfunção endotelial, o aumento da reabsorção de sódio nos rins e a piora no relaxamento do endotélio vascular (ABDULLA, 2011).

Rosengren (2004) mostrou que o estresse é um fator de risco para o desenvolvimento de doenças cardiovasculares nos seres humanos, principalmente infarto agudo do miocárdio, e que essa relação entre o estresse e o aparecimento de doenças cardiovasculares independe da condição socioeconômica e é similar entre homens e mulheres, entre pessoas de diferentes idades, e em todas as regiões do mundo. É notório e bem documentado que a exposição ao estresse evoca o aumento da PAM e da FC em humanos (HUANG, 2013) e em modelos animais (TAVARES e CORRÊA, 2006), além de alterar o controle barorreflexo da FC (CRESTANI, 2010), e aumentar a temperatura em leitos específicos (VIANNA e CARRIVE, 2005).

No contexto da SMet, estudo recente mostra que ratos alimentados com uma dieta hiperlipídica manifestam reatividade cardiovascular aumentada ao estresse emocional (ABREU et al., 2014). Sabe-se que o consumo de frutose, de forma aguda ou crônica, provoca maior simpatoexcitação, e que esta exerce um papel fundamental na SMet induzida por frutose (ABDULLA, 2011). No entanto, alterações na resposta cardiovascular ao estresse em função da dieta rica em frutose ainda não foram elucidadas. Diante o exposto, a hipótese do presente estudo é de que a condição de SMet induzida pela dieta alimentar rica em frutose exacerbe a resposta cardiovascular ao estresse emocional agudo.

\section{OBJETIVOS}

Avaliar os parâmetros cardiovasculares basais, o controle barorreflexo da frequência cardíaca e a resposta cardiovascular ao estresse emocional agudo em ratos com suplementação alimentar rica em frutose.

\section{METODOLOGIA}

Animais - Foram utilizados XX ratos Wistar (4 semanas de idade) provenientes do Biotério Central da Universidade Federal de Alagoas. Os animais foram acomodados em sala apropriada com exaustor de ar, temperatura controlada $\left(22-24^{\circ} \mathrm{C}\right)$ e ciclo claro-escuro de $12-12 \mathrm{~h}$ (06:00h-18:00h), em caixas de polipropileno apropriadas para roedores (3-4 animais/ caixa), com livre acesso à ração padrão para roedores, e ingestão hídrica de água potável ou solução de frutose. Os animais foram pesados semanalmente, bem como foi estimado o consumo médio hídrico (ml) e de ração (g) (média de consumo/ animal/ caixa/ semana).

A partir da $7^{\mathrm{a}}$ semana de idade, os animais receberam solução de frutose $10 \%$ (Synth), preparada em água potável por 10 semanas ad libitum. A solução de frutose foi preparada a cada $48 \mathrm{~h}$ e renovada aos animais.

Canulação de artéria e veia femoral - Sob anestesia da associação de Xilazina (7,5 $\mathrm{mg} / \mathrm{kg}$ ) e Ketamina (80 mg/kg) os animais foram submetidos à canulação de artéria e veia femoral 24h antes do final do experimento para a realização do registro de parâmetros cardiovasculares e administração de substâncias vasoativas e anestésico para eutanásia, respectivamente.

Registro de parâmetros cardiovasculares - Os animais foram submetidos ao registro da PAM e da FC por meio de um transdutor de pressão conectado à cânula da artéria femoral e ao sistema de aquisição de dados Power Lab/ LabChart Pro. A sensibilidade do controle barorreflexo da FC foi avaliada de acordo com a variação reflexa de FC ao aumento da PAM induzido pela administração endovenosa (bolus) de doses crescentes do $\square$-adrenérgico Lfenilefrina $(1,0-20 \quad \mathrm{~L}) \mathrm{g} / \mathrm{nA}$ resposta cardiovascular ao estresse agudo moderado foi avaliada pelas variações de PAM e FC ao estresse por contenção em aparato cilíndrico de acrílico por 20 min. O tônus simpático vascular foi avaliado, pela análise das alterações de PAM 
e FC à administração endovenosa do antagonista -adrenérgico prazosina (0,1 mg/ kg). Em seguida, os animais foram eutanasiados por sobredose anestésica.

\section{RESULTADOS E DISCUSSÃO}

\section{Ganho de peso corporal e ingestão alimentar}

A ingestão hídrica média diária de cada animal mostra que o grupo experimental frutose (FF) apresentou uma tendência de aumento na ingestão em relação ao grupo controle (CTL) ao longo das 10 semanas de estudo (Figura 1A). Quanto à ingestão de ração, o consumo do grupo FF foi menor que o observado no grupo CTL (Figura 1B). Já na primeira semana de ingestão de frutose houve uma tendência à redução da ingestão de ração no grupo FF, resultado esperado devido ao consumo calórico pela ingestão hídrica de solução de frutose $10 \%$ por esses animais. O ganho de peso foi similar entre os grupos (Figura 2).

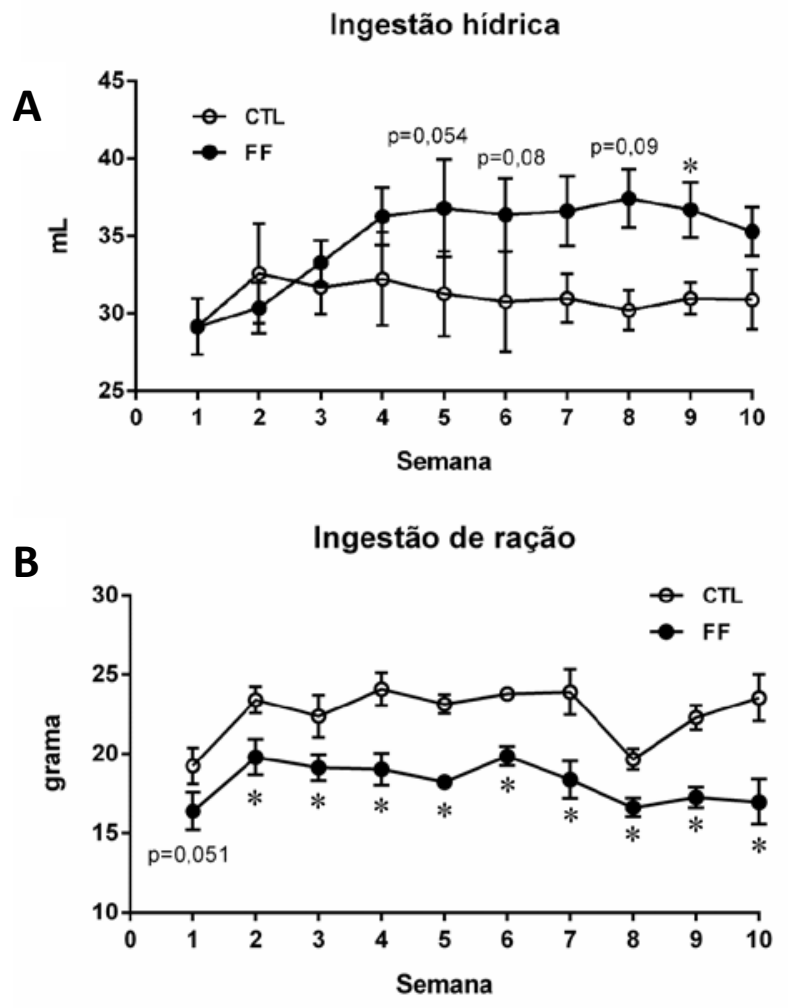

Figura 1. Ingestão hídrica (A) e de ração (B) de ratos Wistar submetidos à ingestão crônica de solução de frutose $10 \%$ por 10 semanas. $\mathrm{CTL}=$ grupo controle ( $\mathrm{n}=3$ caixas; 11 animais); FF $=$ grupo frutose $(\mathrm{n}=3$ caixas; 9 animais). $* \mathrm{p}<0,05$ vs CTL; Two-way ANOVA medidas repetidas/ Newman-Keuls. 


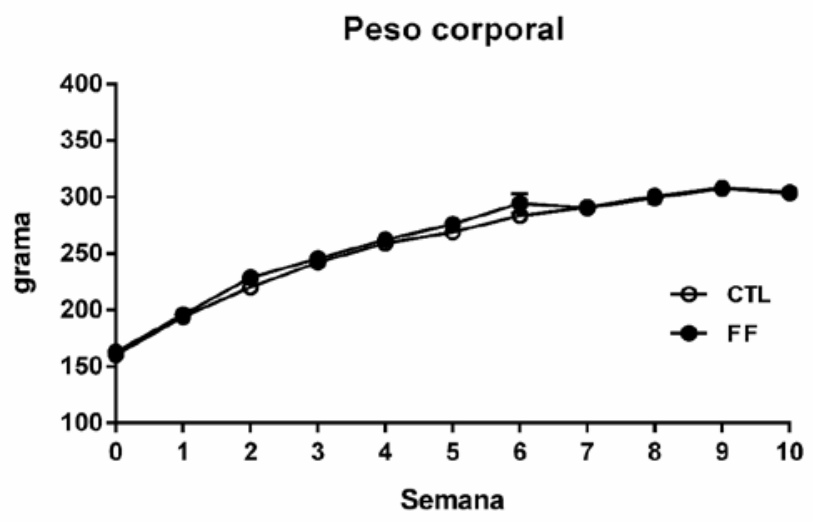

Figura 2. Peso corporal de ratos Wistar submetidos à ingestão crônica de solução de frutose $10 \%$ por 10 semanas. CTL $=$ grupo controle $(n=11$ animais $) ; \mathrm{FF}=$ grupo frutose $(\mathrm{n}=9$ animais $)$.

\section{Parâmetros cardiovasculares basais}

Os ratos que foram submetidos à suplementação alimentar crônica de solução de frutose $(100 \mathrm{~g} / \mathrm{L})$ por 10 semanas (grupo FF) apresentaram aumento significativo na PAM quando comparados ao grupo controle $(\mathrm{CTL}=117,14 \pm 1,5 \mathrm{mmHg} v s \mathrm{FF}=129,71 \pm 2,0 \mathrm{mmHg}$, $p<0,05$; Figura 03A). Em relação à frequência cardíaca (FC), foi observado um aumento discreto no grupo FF em relação ao CTL, havendo uma tendência da diferença ser estatisticamente significativa (CTL $=287,29 \pm 8,7 \mathrm{bpm} v$ sF $=309,86 \pm 7,1 \mathrm{bpm} ; p=0,067$; Figura 03B).

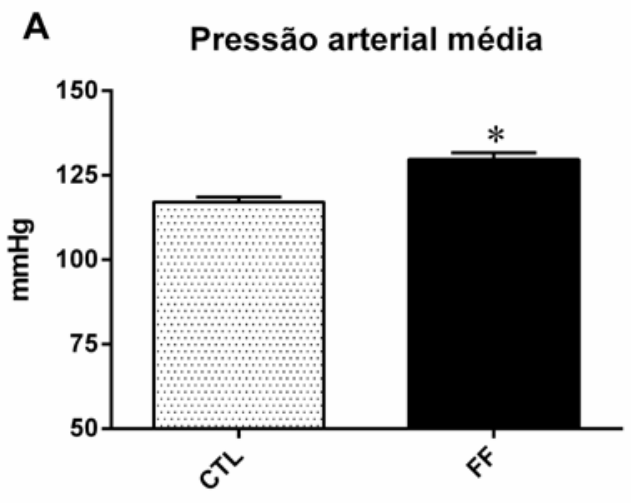

B

Frequência cardíaca

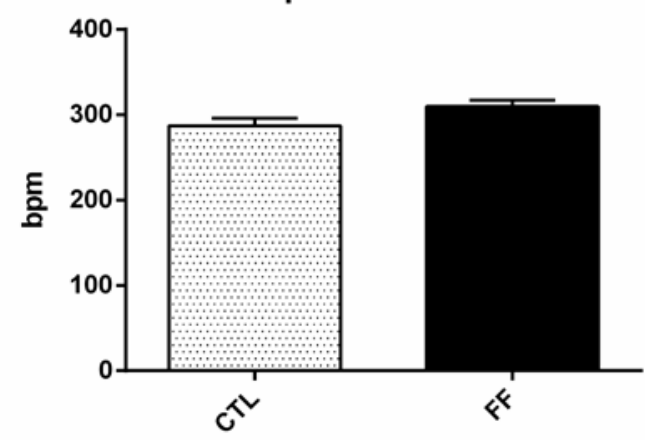

Figura 3. Pressão arterial média (A) e frequência cardíaca (B) basais de ratos Wistar submetidos à ingestão crônica de solução de frutose $(100 \mathrm{~g} / \mathrm{l})$ por 10 semanas. $\mathrm{CTL}=$ grupo controle $(\mathrm{n}=7)$; $\mathrm{FF}=$ grupo frutose $(\mathrm{n}=7) .{ }^{*} \mathrm{p}<0,05$ vs CTL, teste t-Student não pareado. 


\section{Controle barorreflexo da frequência cardíaca}

Também foi observado que os ratos do grupo FF, apresentaram um prejuízo no índice de sensibilidade do controle barorreflexo da FC em relação ao grupo CTL (CTL = $1.620 \pm 0.08$ $\mathrm{ms} / \mathrm{mmHg}$ vs FF $=1.110 \pm 0.08 \mathrm{~ms} / \mathrm{mmHg} ; p<0,05$; Figura 04A). Este resultado mostra que 0 grupo que recebeu a suplementação de frutose apresenta um prejuízo na resposta bradicárdica ao aumento agudo da PAM em relação ao grupo CTL. Tais alterações também podem ser observadas pela menor inclinação da reta que correlaciona as variações médias de FC frente às variações médias de PAM para o grupo FF, quando comparada ao grupo CTL (Figura 04B).

\section{A}

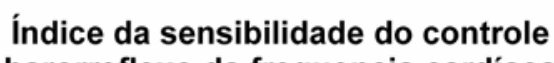
barorreflexo da frequencia cardíaca

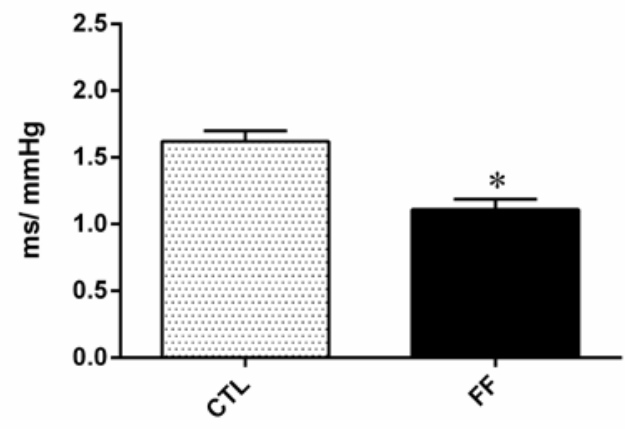

B

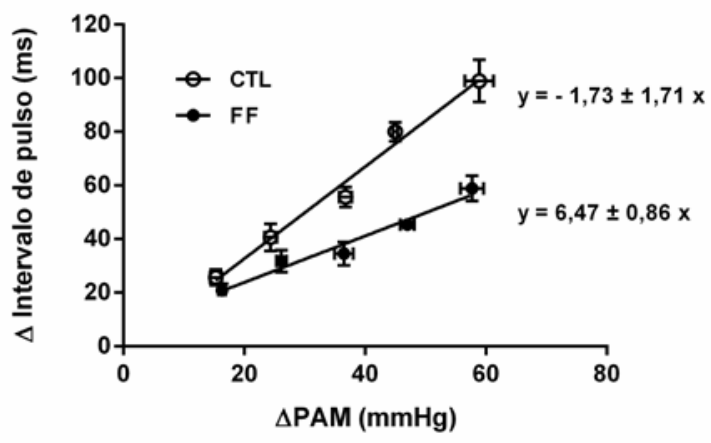

Figura 4. Índice de sensibilidade do controle barorreflexo da FC (A) e ilustração das alterações médias no intervalo de pulso frente às variações médias de PAM (B) de ratos Wistar submetidos à ingestão crônica de solução de frutose $(100 \mathrm{~g} / \mathrm{l})$ por 10 semanas. CTL = grupo controle $(n=7)$; FF = grupo frutose $(n=6)$. $* \mathrm{p}<0,05$ teste t-Student.

\section{Resposta cardiovascular ao estresse emocional agudo}

Tal como observado por outros grupos de pesquisa, a contenção induziu um aumento significativo da PAM e da FC, alterações típicas da resposta cardiovascular ao estresse. Em particular, o presente estudo estende os achados da literatura mostrando que a suplementação alimentar crônica de frutose contribuiu para uma resposta cardiovascular exacerbada, uma vez que ratos do grupo FF apresentaram curvas de variação pressórica e taquicárdica maiores quando comparados aos animais do grupo CTL, se estendendo ao período de recuperação (Figuras 5 e 6).

A intensidade das variações dos parâmetros cardiovasculares varia de acordo com os diferentes tipos de agentes estressores, e são poucos os dados na literatura científica que relacionam a SMet e a resposta cardiovascular ao estresse agudo, e se isso implica em maior risco ao desenvolvimento de doenças cardiovasculares na condição de SMet. Para o nosso 
conhecimento, nosso estudo é o primeiro a tentar elucidar alterações nas respostas cardiovasculares ao estresse agudo na condição de SMet por suplementação alimentar rica em frutose. Nosso grupo pretende avançar, em estudos futuros, na elucidação dos mecanismos centrais envolvidos com tais alterações.

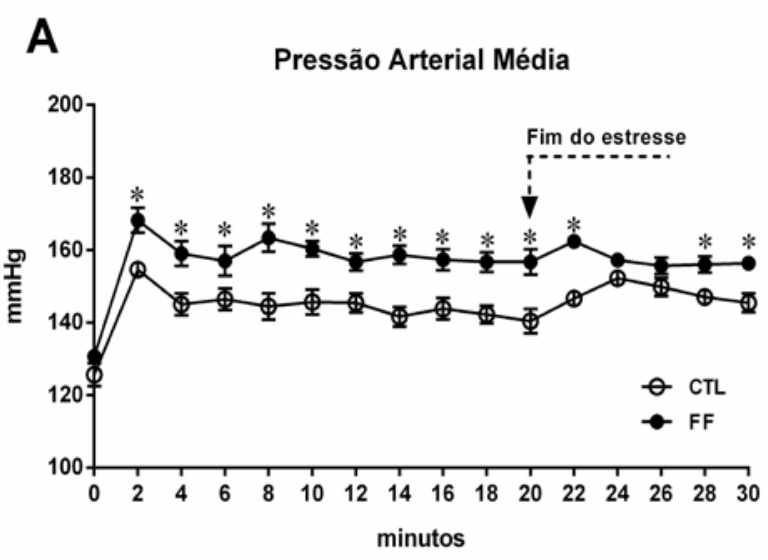

B
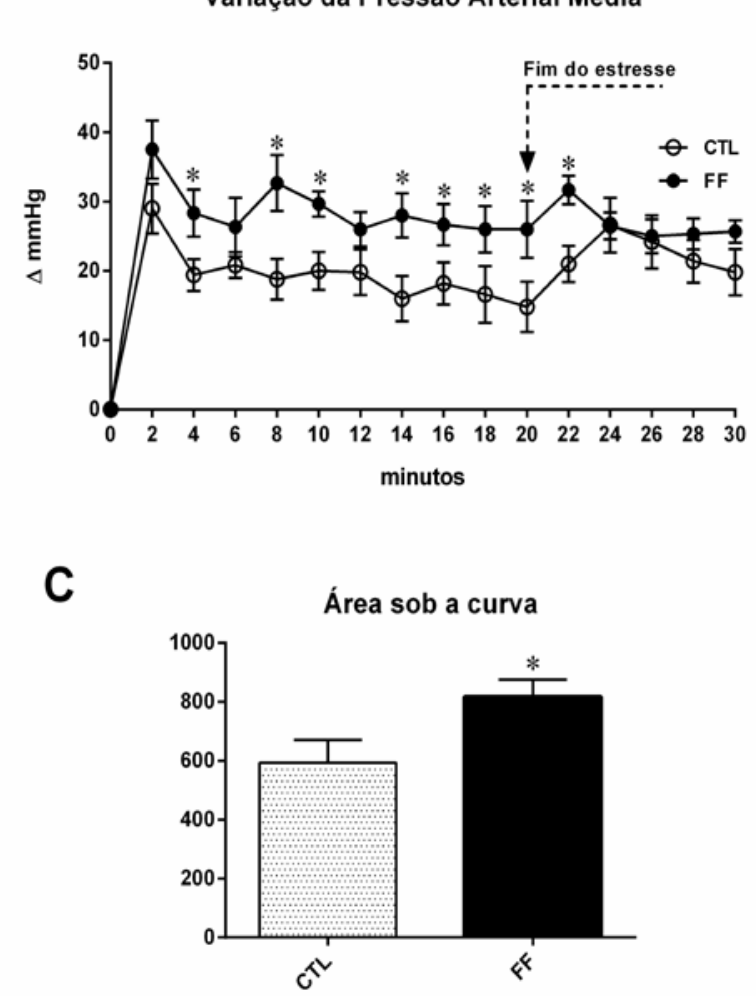

Figura 5. Resposta pressórica ao estresse emocional agudo por contenção em ratos Wistar submetidos à ingestão crônica de solução de frutose $(100 \mathrm{~g} / \mathrm{l})$ por 10 semanas. Valores absolutos de pressão arterial média (PAM; A), variações da PAM (B) e área sob a curva de variação da PAM (C). CTL = grupo controle $(n=7) ; \quad F F=$ grupo frutose $(n=6) .{ }^{*} \mathrm{p}<0,05$ vs CTL, Two-way ANOVA com medidas repetidas/ Newman-Keuls (A e B), test t-Student não pareado (C). 


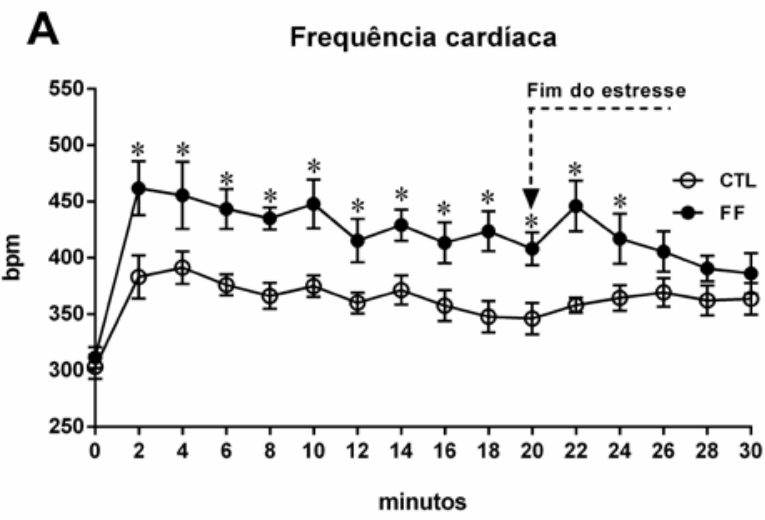

B
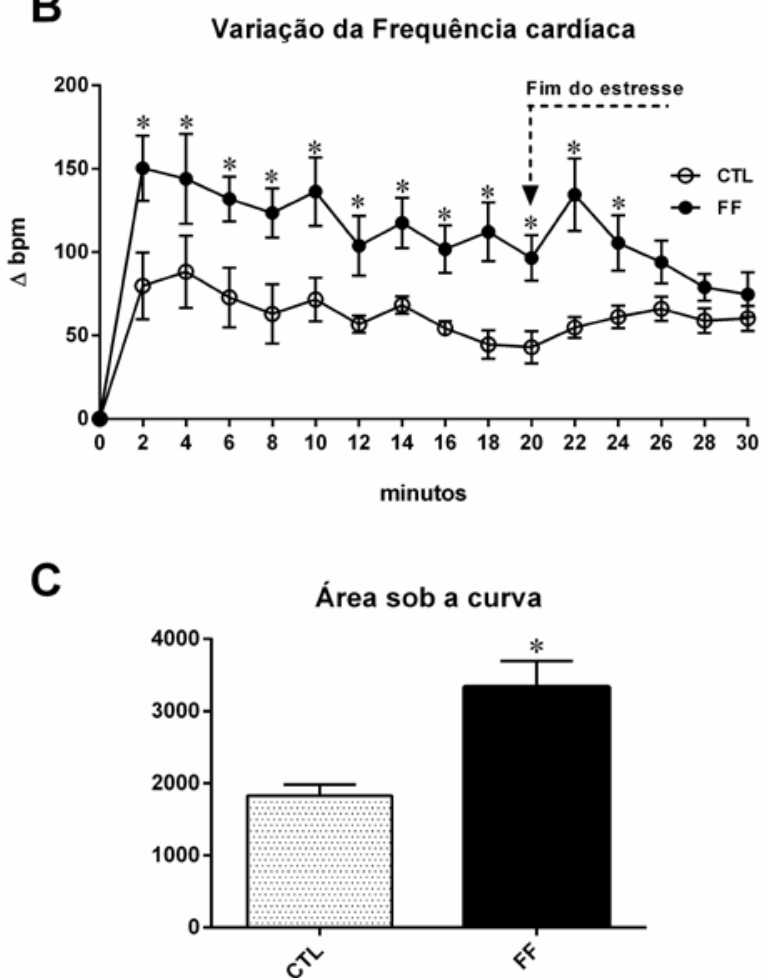

Figura 6. Resposta taquicárdica ao estresse emocional agudo por contenção em ratos Wistar submetidos à ingestão crônica de solução de frutose $10 \%$ por 10 semanas.. Valores absolutos de frequência cardíaca (FC; A), variações da FC (B) e área sob a curva de variação da FC (C). CTL = grupo controle $(\mathrm{n}=7)$; $\mathrm{FF}=$ grupo frutose $(\mathrm{n}=6)$. $* \mathrm{p}<0,05$ vs CTL, Two-way ANOVA com medidas repetidas/ NewmanKeuls (A e B), test t-Student não pareado (C).

\section{Tônus simpático vascular}

Com relação ao bloqueio farmacológico da atividade simpática vascular, variações de PAM (FF = - 39,67 \pm 4,0 mmHg vs. CTL = - 33,43 $\pm 2,0 \mathrm{mmHg}$; Figura 07A) e de FC (FF = $151,67 \pm 11,2 \mathrm{bpm} v s$. CTL $=125 \pm 12,6 \mathrm{bpm}$; $p>0,05$; Figura 07B), foram similares entre os grupos FF e CTL, sugerindo que a dieta rica em frutose não induziu um aumento no tônus simpático vascular destes animais.

Considerando que uma maior atividade simpática vascular tem sido reportada na literatura científica, nosso resultado segue de encontro a estes achados (BUNNAG., 1997; 
CASTRO, 2015). Uma possível explicação para essa divergência são as diferentes metodologias utilizadas, limitando as conclusões aqui apresentadas.
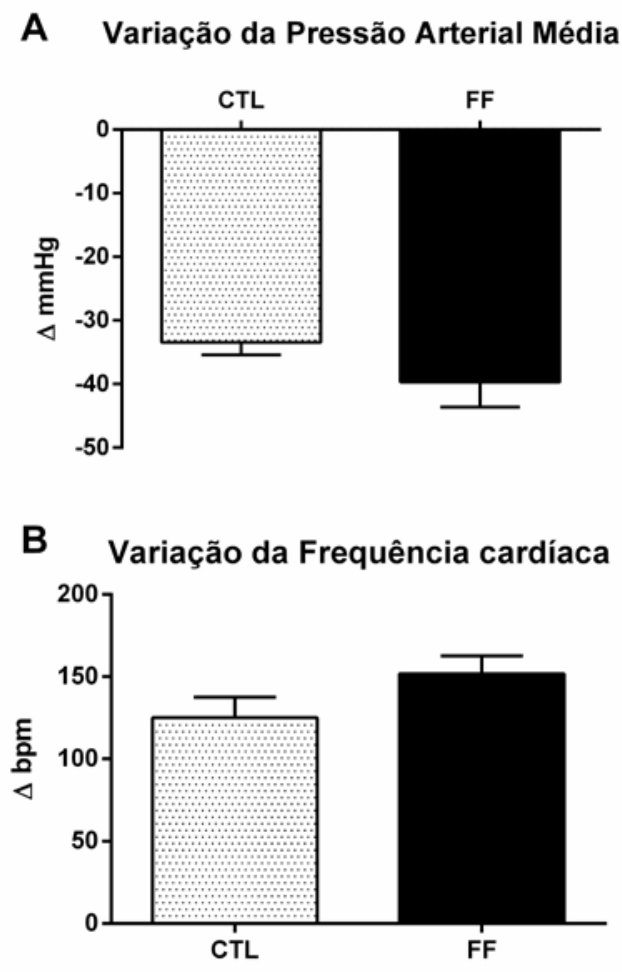

Figura 7. Índice das variações médias da PAM (A) e da FC (B), provocadas pelo bloqueio farmacológico do tônus simpático vascular, de ratos Wistar submetidos à ingestão crônica de solução de frutose $(100 \mathrm{~g} / \mathrm{l})$ por 10 semanas. CTL = grupo controle $(n=7) ; F F=$ grupo frutose $(n=6)$.

\section{CONCLUSÕES}

O estresse é um fator de risco para o desenvolvimento de doenças cardiovasculares, e acomete os seres humanos de forma independe da condição socioeconômica, do gênero e idade. Em adição, a condição de SMet se tornou um tema crítico, de interesse mundial, em função de sua alta prevalência. Em particular, o consumo aumentado de frutose na dieta alimentar humana se apresenta como um potencial fator predisponente ao desenvolvimento de SMet. O presente estudo estende os achados da literatura, mostrando que a suplementação alimentar rica em frutose, além de induzir um aumento da pressão arterial de repouso e prejuízo no controle barorreflexo da frequência cardíaca, induz uma resposta pressórica e taquicárdica exacerbada ao estresse emocional agudo moderado, conferindo a este um potencial fator de risco adicional para o desenvolvimento de doenças cardiovasculares na condição de SMet induzida pelo consumo em excesso deste monossacarídeo.

\section{AGRADECIMENTOS}

Os autores agradecem à Fundação de Amparo à Pesquisa do Estado de Alagoas (FAPEAL) pelo auxílio financeiro, concedido sob a forma de bolsa de iniciação científica a Breno T. G. Fonseca e Bianca M. V. Vasconcelos, pelo Programa de Bolsas de Iniciação Científica da Universidade Federal de Alagoas (PIBIC/ UFAL 2015-2016). 


\section{REFÊRENCIAS}

ABDULLA, M. H.; SATTAR, M. A.; JOHNS, E. J. The Relation between Fructose-Induced Metabolic Syndrome and Altered Renal Haemodynamic and Excretory Function in the Rat. Int. J. Nephrol., v. 2011, Artilce ID 934659, 2011. Disponível em: $<$ http://www.ncbi.nlm.nih.gov/pmc/articles/PMC3139200/pdf/IJN2011-934659.pdf>. Acesso em: 23 mar. 2016.

ABREU, A. R. Blunted GABA-mediated inhibition within the dorsomedial hypothalamus potentiates the cardiovascular response to emotional stress in rats fed a high-fat diet. Neuroscience, v. 262, p. 21-30, 2014.

BUNNAG, P.; HORI, M. T.; ORMSBY, B.; BERGER, M. E.; GOLUB, M. S.; TUCK, M. L. Impaired in vivo adrenergic responses in diet-induced hypertensive rats. Hypertens Res., v. 20, p. 17-21, 1997. http://doi.org/10.1291/hypres.20.17

CRESTANI, C. C. Effect of acute restraint stress on the tachycardiac and bradycardiac responses of the baroreflex in rats. Stress., v. 13, n. 1, p. 61-72, 2010.

CASTRO, E. F. S.; MOSTARDA, C. T.; RODRIGUES, B.; MORAES-SILVA, I. C.; FERIANI, D. J.; DE ANGELIS, K.; IRIGOYEN, M. C. Exercise training prevents increased intraocular pressure and sympathetic vascular modulation in an experimental model of metabolic syndrome. Braz. J. Med. Biol. Res., v. 48, n. 4, p. 332-338, 2015. http://dx.doi.org/10.1590/1414-431X20144217

HUANG, C.-J.; WEBB, H. E.; ZOURDOS, M. C.; ACEVEDO, E. O. Cardiovascular reactivity, stress, and physical activity. Frontiers in Physiology, v. 4, p. 1-13, 2013. http://dx.doi.org/10.3389/fphys.2013.00314

ROSENGREN, A.; HAWKEN, S.; ÔUNPUU, S.; SLIWA, K.; ZUBAID, M.; ALMAHMEED, W. A.; BLACKETT, K. N.; SITTHI-AMORN, C.; SATO, H.; YUSUF, S. Association of psychosocial risk factors with risk of acute myocardial infarction in 11119 cases and 13648 controls from 52 countries (the INTERHEART study): case-control study. Lancet, v. 364, p. 953-962, 2004. http://dx.doi.org/10.1016/S0140-6736(04)17019-0

TAPPY, L.; LÊ, K.A. Metabolic Effects of Fructose and the Worldwide Increase in Obesity. Physiology Reviews, v. 90, p. 23-46, 2010.

TAVARES, R. F.; CORRÊA, F. M. Role of the medial prefrontal cortex in cardiovascular responses to acute restraint in rats. Neuroscience, v. 143, p. 231-240, 2006.

VIANNA, D. M.; CARRIVE, P. Changes in cutaneous and body temperature during and after conditioned fear to context in the rat. Eur. J. Neurosci., v. 21, p. 2505-2512, 2005. 